\title{
Topology optimization and analysis for double acting compliant retractor mechanism
}

\author{
Sonali S. Patil ${ }^{*}$, Sham H. Mankar ${ }^{\dagger}$ and Sandip A. Kale ${ }^{\dagger}$ \\ ${ }^{\dagger}$ Mechanical Engineering Department, Trinity College of Engineering and Research, Savitribai Phule Pune University, Pune, India
}

Accepted 01 Jan 2017, Available online 02 Jan 2017, Vol.7, No.1 (Feb 2017)

\begin{abstract}
Special standards are necessary regarding the drive and motion system in precision engineering, biomechanics application, micro-electro mechanical system (MEMS), nanotechnology application. With the help of conventional rigid body mechanism, backlash problem becomes a more dominant problem in designing a high precision positioning stage with micro-scale (micrometer or nanometer) resolution. Compliant mechanisms are found to be more convenient over rigid body mechanisms. A compliant mechanism is a mechanical device in which elastic deformation is used to achieve force and motion transmission. Compliant mechanism provides a jointless mechanism eliminating issues regarding wear, lubrication, backlash, assembly. The use of compliant mechanism in linear displacement application is investigated in this research. The topology for the mechanism is optimized and the total deformation is calculated with ANSYS software. The software results are checked by performing actual experimentation. Therefore compliant retractor mechanism is very well suited for positioning by scale up the motion of linear stage and topology optimization, analyze and test a compliant retractor mechanism.
\end{abstract}

Keywords: Compliant mechanism, Elastic deformation, Topology, Topology Optimization.

\section{Introduction}

A mechanism is nothing but a mechanical device to transfer motion, energy of force. A rigid body mechanism consists of rigid links and joints for the purpose. Conventional rigid body mechanisms are usually assembled from discrete components and designed to be strong and stiff. These rigid body mechanisms transfer motion through their rigid links and rigid joints because of which mechanisms have several shortcomings such as backlash, wear, requirement of lubrication, less accuracy. A compliant mechanism is defined as single piece flexible structure which uses elastic deformation to achieve force and motion transmission from one place to another.

The primary ways of creating flexibility are material properties, geometry, loading and boundary conditions. Compliant mechanisms offer a jointless alternative over conventional rigid body mechanisms eliminating issues of friction and backlash and important maintenance. Structures in precision applications have to be monolithic and assembly must be avoided due to limitations of microfabrication methods. Hence it can often compact and reduces cost of parts material and assembly of the components. Many emerging applications like precision application, micro-electromechanical systems (MEMS) are made

*Corresponding author: Sonali S. Patil attractive with the absence of hinges or joints (Howell, 2009).

Rhombic micro-displacement amplifier for piezoelectric actuator was proposed by J. Chen, C. Zhang. First geometric amplification relations are analyzed and linear model is built to analyze the electrical and properties of this amplifier then accurate modeling method of amplifier is studied for important amplification (Chen et. al., 2014).

A methodology for designing compliant mechanism with piezoelectric actuation to obtain maximized deflection and force at output is proposed by Bharti and Frecker. The focus is on design of compliant mechanism with multiple optimally placed and sized piezoelectric actuators (Bharti \& Frecker, 2003).

New topology that is a symmetric mechanism for displacement amplification and compliant mechanical amplifier based on new topology is designed to amplify the stroke of piezoelectric actuator was proposed by Ouyang et.al in 2005.

Compliant mechanical amplifier based on a symmetric compliant mechanism was studied for performance optimization was previously proposed by Acob et. al in 2012. The amplifier was studied for performance optimization and the amplifier was optimized based on its most significant design parameters optimized over a range of input displacements and various load cases with goals of large amplification ratio and high natural frequency. 
Use of pseudo rigid body model for designing compliant mechanism is advocated by Baker and Howell. The method of analysis shows the large deflection to be modeled using rigid body kinematics simplifying the design of compliant mechanisms (Piang, 2001).

The design theory and synthesis of compliant microleverage mechanisms including single stage microleverage mechanism is presented by Xiao-Ping S. and Henry S. Yang as the building block for multistage microleverage mechanisms (Singh et.al., 2013).

Motion analysis of 1 degree of freedom pantograph based micro-nano scratching machine has been performed by S. K. Singh et.al. to ensure transferring of actuated micro nano scale linear motion to tool tip in a single axis to have better control over depth of cut . Also the investigation shows potential reduction of linear motion by upto $1 / 4$ th of riving point displacement. The methods for parameterizing the design domain i.e. ground structure and continuum approaches, are independent of the optimization problem formulations. The choice of parameterization is made by the designer and primarily on computation time and software availability (Bendsoe, 2003). The ground structure method may be preferred when computation time is important because a relatively small number of elements can be used and because a relatively easy to develop the finite analysis code (Pederson). The homogenization method is more complicated and requires more computation time, and the homogenization formulas may not be available to the average designer trying to implement their own code. The SIMP method is convenient for many problems, in part due to the availability of the software on the Top Opt website. The designer should understand underlying problem formulation and parameterization and any associated assumptions and limitations of these.

The topology optimization is nothing but pattern of connecting sequential members method in a design domain subjected to loading and boundary conditions; such that the compliance of structure is minimized (Ananthasuresh et. al., 2001). ESO method can easily be extended to $3 \mathrm{D}$ and more complex compliant mechanisms specifications like thermally or electro thermally actuated structures especially since the computations very tractable. The application of the topology optimization model in various fields of engineering can significantly improve quality which is important in global competition (Ananthasuresh et. al., 2001). A newly developed qp-relaxation for element connectivity parameterization is achieved stress-based topology optimization for geometrically nonlinear structures by Moon \&Yoon in 2013. Robust design of large displacement compliant mechanisms is achieved by Lazarov et. al. in 2011. Based on combination of topology optimization and optimal control method freedom of engineering design is enlarged and the designer can give more reasonable design to cater for engineering requirement. The computational issues stress measure, the number of stress evaluation points inside an element, and correction parameter for a stable topology optimization process,. A reduction strategy on target reliability index is proposed and utilized together with the strain relaxation approach. Topology optimization has industrial applications such as design of automotive and airplane structures. Mechanical compliant retractor is used to amplify input displacement to carry out non conventional machining process.

\section{Methodology}

First select the design domain and apply appropriate loading and boundary conditions to it. Find the optimized topology solution for the same. The total deformation is achieved with ANSYS software and is checked with actual experimentation result. The methodology is described in Fig.1.

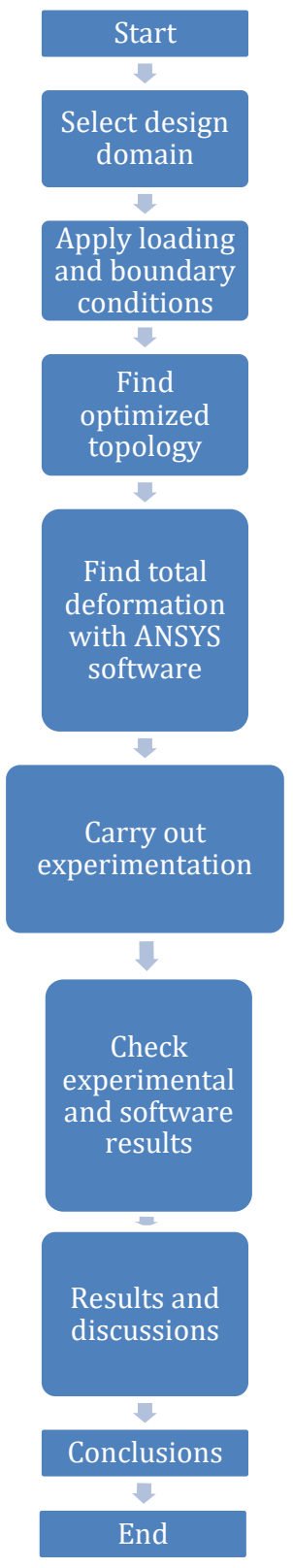

Fig.1 Flowchart of the methodology 


\section{Toplogy optimization}

\subsection{Computational}

To find topology optimized solution for compliant retractor mechanism.

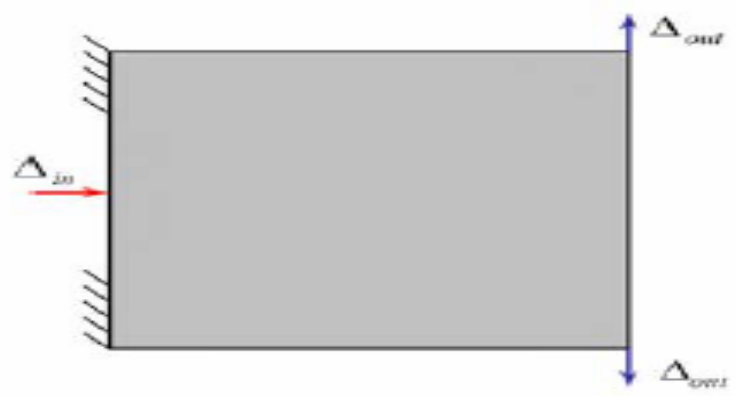

Fig.2 Problem Fig

The topology optimization problem of distributing a given amount of material in a design domain is subjected to loading and boundary conditions such that compliance of structure is minimized. Some particular areas in the design domain can be specified as of solid materials (black areas) and some areas can be specified to be void (white areas). The rectangular design domain is discretized by $\mathrm{N}$ number of finite square elements.

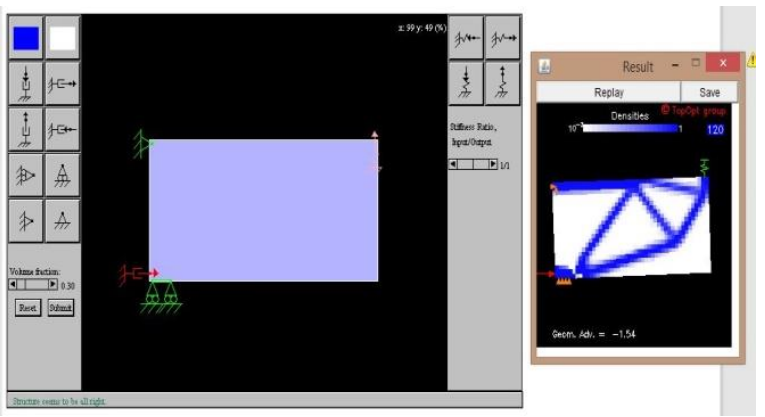

Fig 3.Topology Optimized Solution

The optimized topology solution is obtained with the help of Top0pt software by applying suitable boundary and loading conditions. The design domain represents half symmetric portion of the design domain. The boundary appropriate conditions are two rigid supports at left vertical edge of design domain.

\subsection{Experimentation}

The compliant retractor mechanism of $60 \mathrm{~mm} \mathrm{X} 40 \mathrm{~mm}$ dimension is manufactured with the help of laser beam machining. The stainless steel material is selected because of its easy availability and ductility. Some short portion is extended for the fixing of mechanism with alen bolts.

Figure 4 shows the experimental set up for compliant retractor mechanism. The input loading is in horizontal direction at the middle edge. Output displacement is measured at output tongs.

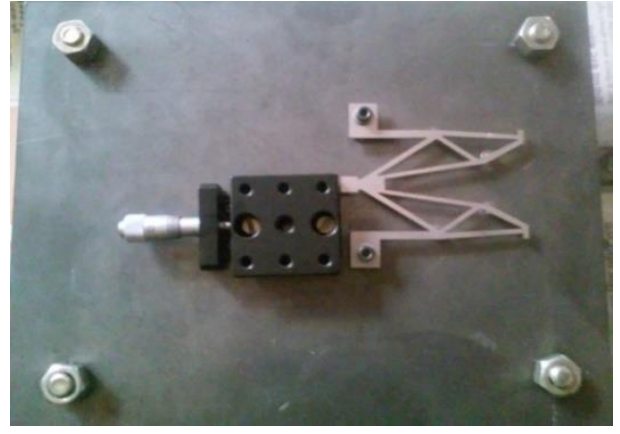

Fig. 4. Photogragh of experimental set up

The input displacement is given by micro actuator. The output displacement is measured at output end by clicking photograph before actuation and after actuation and importing the same pictures in AutoCAD and dimensioning them. We can find the difference in between both and find the net output displacement.

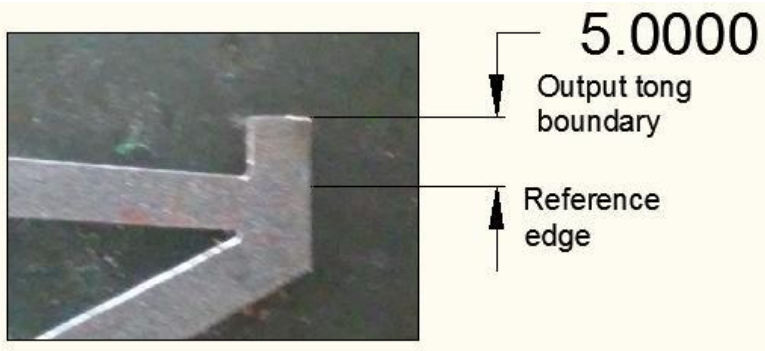

(a)

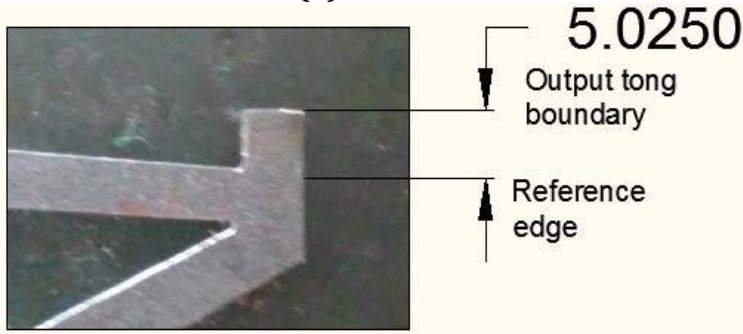

(b)

Fig.5 (a) Measurement before actuation, (b) Measurement after actuation

The input is displacement is given in the range of 10 micrometer to 50 micrometer. The deformation is measured with the help of AutoCAD software. Fig 5. (a) and (b) shows measurement before actuation and after actuation respectively. The difference in two reading gives displacement at output of retractor mechanism.

\section{Analysis of complaint retractor mechanism}

The modeling of the above topology optimized compliant retractor is done with the help of ANSYS software. ANSYS 15.0 software is used and virtual model of the compliant retractor is done considering all dimensions. Fig. 6. shows $3 \mathrm{D}$ model of compliant retractor mechanism. 


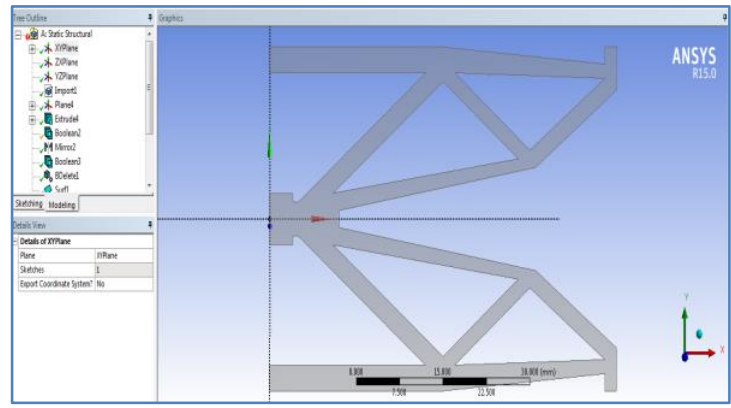

Fig. 6. 3 D model of compliant retractor mechanism

Figure 7. shows the 3D model of the compliant retractor with $\mathrm{X}, \mathrm{Y}, \mathrm{Z}$ axis orientation and proper loading and boundary conditions. $X$ axis is having longitudinal i.e. length which is equivalent to $60 \mathrm{~mm}$. Y axis consists of width equal to $40 \mathrm{~mm}$ and $\mathrm{Z}$ axis having thickness equal to $1 \mathrm{~mm}$.

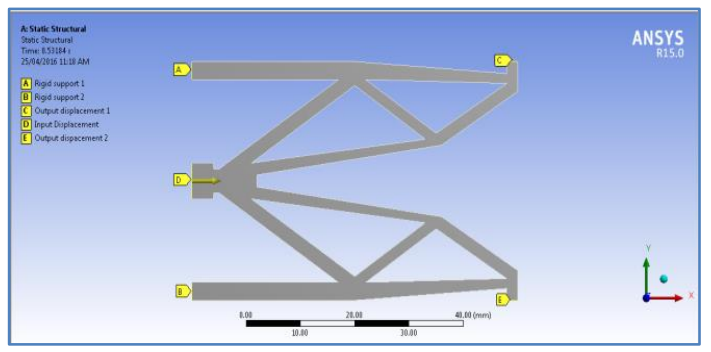

Fig. 7 Loading and Boundary conditions used for analysis

Fig.8. gives information about the total deformation occurs in the compliant retractor mechanism. The maximum deformation is 30.30 micrometer for 10 micrometer input displacement. The compliant retractor mechanism is giving approximately 3 times output displacement as that of input displacement. The mechanism is having amplification ratio as 3.024 .

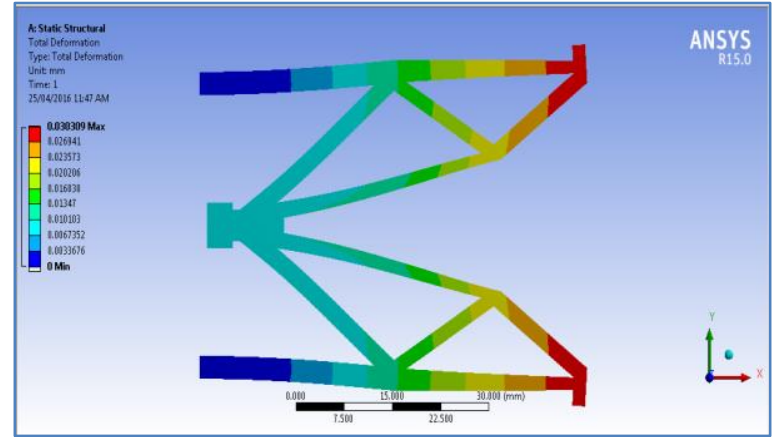

Fig.8 Total deformation distribution in compliant retractor mechanism

It should be noted that all the tables must be firstly drawn in Excel and after that they should be placed in your article. The heading of the table should be above the table. An example is given as under.

\section{Results and discussions}

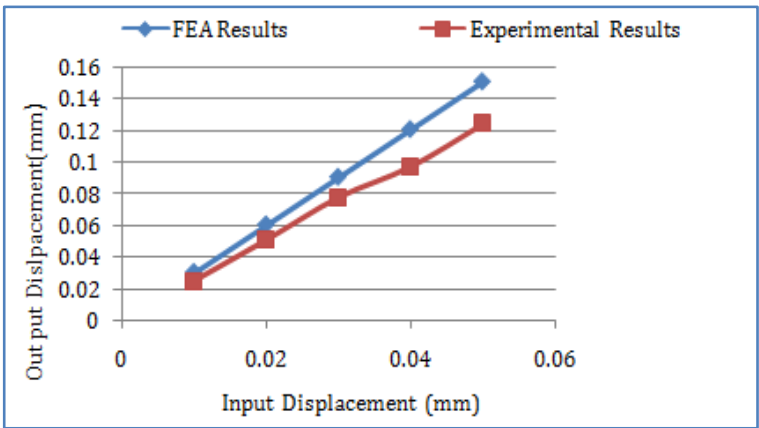

Fig. 9. Comparison between output displacement

As shown in Fig 9, the graph of experimental values of output displacement nearly coincides with analytical values. The deviation in both values may be due to minor friction present between the surface of mechanism and base plate.

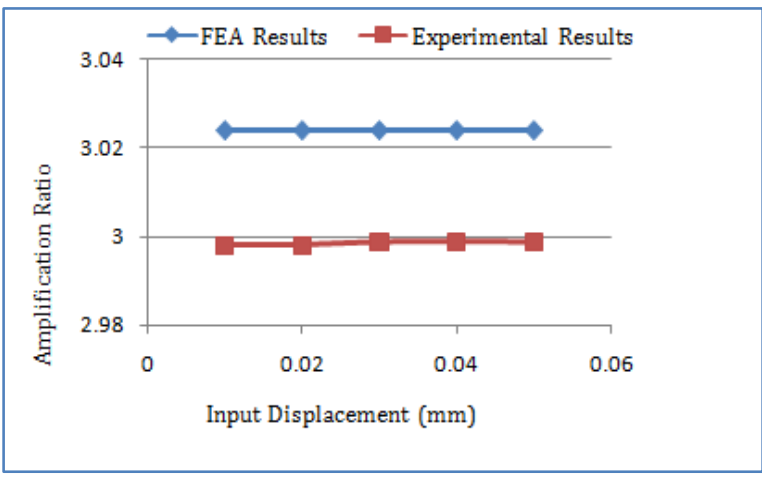

Fig.10 Comparison between amplification ratio

As shown in Fig 10, the graph of experimental values of amplification ratio nearly coincides with analytical values. The deviation in both values may be due to minor friction present between the surface of mechanism and base plate.

\section{Conclusions}

Compliant retractor mechanism can be used for magnification of displacement in precision applications, micro-electromechanical system (MEMS) etc. Due to significant advantages of compliant mechanism over rigid body mechanism, the fabrication of mechanism transmitting micro range displacement is possible. The result shows three times displacement at output of compliant retractor mechanism. Friction between mechanism surface and surface of base plate may cause deviation result from software and actual experimental results.

\section{References}

Ananthasuresh, G. K. and M. Frecker, (2001), Optimal Synthesis with Continuum Models, in Compliant Mechanisms, John Wiley and Sons. 
Andreassen, E., A. Clausen , M. Schevenels, B.S. Lazarov, and 0. Sigmud, (2011), Efficient topology optimization in MATLAB using 88 lines of code. Structural and Mutidisciplinary Optimization, 43(1):p. 1-16.

Bendsoe, M.P. and O C, (2003), Topology optimization: Theory Methods and Applications. Berlin Heidelbgrg: Springer-Verlag.

Bharti Smita and Frecker Mary, (2003), Compliant Mechanical Amplifier Design using Mutiple Optimally Placed Actuators, ASME International Mechanical Engineering Congress, pp.1-8

Jinglong Chen, Chulin Zhang, Minglong $\mathrm{Xu}$, Yanyang zi and Xinong Zhang, (2014), Rhombic Micro-displacement Amplifier For Piezoelectric Actuator and Its Linear And Hybrid Model, Mechanical systems and Signal Processing, pp.1-14

John Michael Acob, Vangjel Pano and Ouyang, P. R., (2012), Optimization of a Compliant Mechanical Amplifier based on a symmetric Five Bar Topology, pp.323-332

L. Howell, Compliant Mechanisms, (2009), New York: John Wiley \& Sons.
Lazarov B.S., M. Schevenels, and O. Sigmund, (2011), Robust design of large displacement compliant mechanisms. Mechanical Sciences.2: p.175-182.

Ouyang P. R., Zhang W.J. and Gupta M. M., (2005), Design of a New Compliant Mechanical Amplifier, ASME international Design Technical Conferences, pp.1-10

Pederson, C.B.W., T. Buhl, and O. Sigmud, (2001), Toplology synthesis of large displacement compliant mechanisms.

Seung Jae Moon, Gil Ho Yoon, (2013), A newly developed qprelaxation for element connectivity parameterization ti achieve stress-based topology optimization for geometrically nonlinear structures, Comput. Method Appl. Mech.Engrg., 265, pp. 226-241.

Shishir Kumar Singh, Soumen Mandal and Nagahanumaiah., (2013), Motion and Force Analysis in Pantograph Mechanisms for Micro-Nano Pattrening , Procedia Engineering, (64), pp 1445-1453, Intrenational,

Xiao-Piang S, Su, and Henry S. Yang., (2001), Design of Compliant Micro leverage Mechanisms, Sensors and Actuators, (A87), pp.146-156 